\title{
Flowers visited by honey bee in southern Finland
}

\author{
Markku Käpylä (1) and PaAvo Niemelä (2) \\ (1) Department of Biology, University of Jyväskylä, SF-40100 \\ Jyväskylä, Finland.
}

(2) Deceased 12. VIII. 1951.

\begin{abstract}
A list of nectar and pollen sources of honey bee (Apis mellifera L.) in southern Finland based on 44500 flower records is presented. Only the common wild and cultivated species are included, 139 species altogether. The flowering times are shown with an accuracy of two weeks.

The most important food sources during spring (April-May) are Salix spp., and Tussilago farfara; during early summer (late May and June) Salix spp., Taraxacum officinale, Acer platanoides, Vaccinium vitis-idaea, Barbarea vulgaris, Ribes spp., Malus domestica, Sorbus aucuparia, and Geranium sylvaticum; during mid-summer Trifolium repens, T. hybridum, Rubus idaeus, Tilia cordata, Epilobium angustifolium, and Cirsium arvense; during late summer and early autumn Calluna vulgaris, Arctium tomentosum, Sonchus arvensis and Leontodon autumnalis.
\end{abstract}

\section{Introduction}

Beekeepers doubtless have a good idea of the flowers visited by their bees. Systematic observations concerning the whole flora are, however, rare in Finland. Martimo (1945) has made pollen analyses of 21 honey samples. The most comprehensive is AARIo's (1962) pollen analysis study of one year's honey production in Finland. There is also data concerning competing flowers in relation to pollination of red clover (SARISAlo and VAlle 1969). Finnish guidebooks for beekeepers (Mali and LaAksonen 1961, KuUrma and PakariNEN 1961) also contain lists of pollen and nectar plants probably based partly on foreign sources, partly on unsystematic observations of the writers.

This paper presents a list of plants visited by honey bees in southern Finland. It is based on a large material of field observations. The list is intended as a basis to be completed later on. Flowering times are also shown. The field observations on flower visiting were made by Paavo Niemelä. The material was rearranged and the paper was written by Markku Käpylä. 


\section{Material}

Field observations were made during the summers $1931-33$ by Paavo Niemelä, mostly in southwestern Finland. Most of the observations were made in Perniö $\left(60^{\circ} 12^{\prime} \mathrm{N}, 23^{\circ} 8^{\prime} \mathrm{E}\right)$ and its neighbouring municipalities Särkisalo, Uskela and Halikko. Another important area of observations was Turku and its surroundings. This material forms part of Niemelä's (1934) M. Sc. thesis. The original material comprises about 44500 records of flower visits of honey bees on 591 species of plants. This long list of plant species included also many species observed in the Botanical Gardens of the University of Helsinki. Species that are not common in Finland either as wild or cultivated are omitted from this publication.

This unpublished M. Sc. thesis was the main work of Paavo Niemelä. It is still one of the best compilations of data on flower visits of bees of a certain area. It was intended to be developed into a $\mathrm{Ph}$. $\mathrm{D}$. thesis, but was never completed. After a serious illness Paavo Niemelä died at the age of 40. An obituary was written by VALle (1951).

\section{Methods}

The observer walked around and noted every flower visit seen. No corrections were made relating to the commenness of the plant species. Each visit of an individual bee to any flower species was recorded as a single observation. Several consecutive visits of the same individual to the same plant species were also recorded as one observation. The exact criteria used by Paavo Niemelä in deciding the reasons for flower visits are not known, but erroneous observations are probably very few, because of Niemelä's wide experience and knowledge on bees. The difficulties in making the correct field observations are discussed by KÄPYLÄ (1978 a).

The flowering times are mostly compiled according to available literature (Reuter 1948, 1952, 1957, Perttula 1952, Hiitonen and Poijärvi 1954, LöNNQvist 1974, KÄPYLÄ 1978 a), partly according to Käpylä's unpublished observations.

The limiting of a flowering period is subjective. Especially the end of flowering is difficult to determine. In this case occasional flowering after the main flowering season is not included (e.g. Taraxacum).

\section{Results}

The results are shown in tabular form (Table 1). Plant names are according to EHRENDORFer (1973), or if not included there, according to Tutin et al. (1964-1976).

Most of the flowers may be divided according to their flowering time into the following categories. (1) Spring flowers, in which the main flowering period is May, partly already late April. In spring species the flowering time may vary considerably from year to year depending on the weather conditions. (2) Early summer flowers, which flower from the end of May for three to four weeks. Spring and early summer species usually have a short and intensive flowering. (3) The mid-summer flowers start their flowering at the end of 
Table 1. Flower species visited by honey bees. Frequency in foraging is given by a scale $1-5$ ( $\mathrm{N}=$ nectar, $\mathrm{P}=$ pollen), based on the amount of flower visits observed:

$1=$ occasional: mean $<4$ records per year.

$2=$ rare: mean $4-10$ records per year.

$3=$ fair: mean $11-20$ records per year.

$4=$ abundant: mean $21-50$ records per year.

$5=$ very adunbant: mean $>50$ records per year.

Flowering times in southern Finland are shown with the accuracy of two weeks. Numbers 4-9 refer to months (April-September).

Species

Flowering time

\begin{tabular}{llllllll}
\cline { 3 - 6 } & $\mathrm{P}$ & 4 & 5 & 6 & 7 & 8 & 9
\end{tabular}

Acer platanoides

Achillea millefolium ..................... 1

A. ptarmica

Allium schoenoprasum ................ 1

Anemone nemorosa ..................... -

Angelica sylvestris .................... 2

Anthriscus sylvestris ................ 1

Arabis alpina ....................... 5

Arctium minus ......................... 1

A. tomentosum ........................... 5

Barbarea vulgaris .................... 5

Berteroa incana ....................... 1

Butomus umbellatus ................... 1

Calendula officinalis .................. 1

Calluna vulgaris ....................... 5

Caltha palustris

Campanula persicifolia ............... 1

C. rapunculoides ........................ 1

C. rotundifolia

Caragana arborescens...................

Caiduus crispus ......................

Carum carvi ............................. -

Centaurea cyanus ........................

C. jacea .................................. 3

C. phrygia ............................. 1

Chelidonium majus .................... -

Cirsium arvense ........................ 5

C. heterophyllum ..................... 1

C. vulgare ............................. 2

Cornus alba ........................... 1

Corydalis solida ........................ 2

Crataegus intricata .................. 11

Crocus napolitanus .................... - 2

Cucumis sativus ..................... 5 -

Echium vulgare ....................... 5

Epilobium angustifolium .............. 5 5 5

Filipendula ulmaria .................. - 1

Fragaria ananassa ..................... $4 \quad 4$

Frangula alnus ........................ 5

Geranium sylvaticum
5

-

$-$

4

2

5

5

$-$

5

$-$




$\begin{array}{llll}6 & 7 & 8\end{array}$

Geum rivale

Glechoma hederacea

Gynanchum vincetoxicum

Hepatica nobilis

Heracleum spondylium

Hieracium pilosella

H. umbellatum

Isatis tinctoria .

Lamium purpureum

Lathyrus odoratus

L. pratensis

Ledum palustre

Leontodon autumnalis

Linaria vulgaris

Lonicera tatarica

Lotus corniculatus

Lupinus polyphyllus

Lychnis viscaria

Lythrum salicaria

Malus domestica

Malva moschata

Melilotus alba

Myosotis sylvatica

Origanum vulgare

Oxalis acetosella

Papaver croceum

Parthenocissus inserta

Pastinaca sativa

Plantago media

Polemonium coeruleum

Polygonatum odoratum

Potentilla anserina

$P$. erecta

$P$. palustris

Prunus avium

P. domestica

P. padus

Pyrus communis

Ranunculus repens

Rheum rhaponticum

Ribes alpinum

R. nigrum

R. rubrum

R. uva-crispa

Rosa pimpinellifolia

Rubus arcticus .

$R$. idaeus

R. saxatilis

Rudbeckia laciniata

Salix alba

\section{2}

1 -

$1-$

$-1$

$1-$

$1-$

$1-$

$2 \quad 2$

$5-$

1 -

1 -

55

$4 \quad 1$

11

53

$1-$

- 5

15

21

55

$2-$

22

$2-$

$4 \quad 4$

11

- 4

55

$1-$

1
$-\quad 1$

11

$1-$

11

1
$-\quad 1$

$3-$

55

55

13

$\begin{array}{rr}5 & 5 \\ 1 & -\end{array}$

- 2

$1-$

$4-$

$4-$

52

5
$-\quad 4$

11

55

$3-$

$2-$

$\begin{array}{ll}2 & - \\ 5 & 5\end{array}$

$$
\begin{aligned}
& ----+++---- \\
& ----++---\cdots \\
& -----++++-- \\
& -+++------ \\
& -----+++-1 \\
& ----+++---- \\
& ------++++- \\
& ----+++--- \\
& ---+++++++++ \\
& -------++++ \\
& -----++(+)(+)(+)-- \\
& ----++----\cdots \\
& ------+++++ \\
& ------+++-\cdots \\
& ----++----\cdots \\
& ---+++++++- \\
& -----++++-\cdots \\
& ----+++---\cdots \\
& -----++++- \\
& ---++---\cdots+ \\
& ------+++-- \\
& ------++++-\cdots \\
& ----++++++-\cdots \\
& ------+++-- \\
& ---+++---\cdots \\
& -----++++-- \\
& -----+++-\cdots \\
& ------+++-- \\
& ----++++--\cdots \\
& -----++++-- \\
& ---++----\cdots \\
& ----+++++-- \\
& ---++++++++- \\
& ----1++--- \\
& ---++----\cdots \\
& ---++----- \\
& ---++----- \\
& ---++----- \\
& ----(+)++(+)(+)(+)-- \\
& ----++--- \\
& ---++----- \\
& ---++----- \\
& ---++---- \\
& ---++---- \\
& -----++--- \\
& ----++---- \\
& -----++--- \\
& ----++----
\end{aligned}
$$

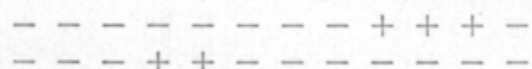

Continued 


N P

\section{4}

5

6

7

8

S. aurita .............................. 14

S. caprea ............................ $5 \quad 5$

S. fragilis ........................... 5

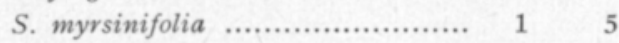

S. phylicifolia ........................... 2 5

S. purpurea ............................ 5

S. repens ............................. 115

Sambucus racemosa ................... - 1

Scilla siberica ......................... - 3

Scrophularia nodosa ................ 1 -

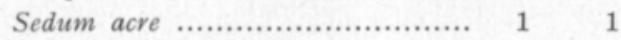

S. aizoon ............................... - 4

S. album ................................. 11

S. hybridum .......................... $4 \quad 1$

S. spurium .............................. $5 \quad 1$

S. telephium ........................... $5 \quad 3$

Sinapis alba ........................... 55

S. arvensis ............................ 1 -

Solanum dulcamara ................... -1

Solidago canadensis .................. $4 \quad 4$

S. virgaurea ........................... 2 2 1

Sonchus arvensis ..................... $4 \quad 2$

Sonchus oleraceus ...................... 31

Sorbaria sorbifolia ..................... 14

Sorbus aucuparia ....................... $54 \quad 5$

Spiraea chameadryfolia ............... 110.5

Stachys byzantina ..................... 5 -

Stellaria graminea ..................... 1 -

Syringa vulgaris ........................ - 4

Tagetes erecta ............................ 2 -

Tagetes patula ......................... 1 -

Taraxacum officinale .................. 55

Thlaspi alpestre ......................... 54

Tymus serpyllum .................... 4 -

Tilia cordata ........................... 5.5

Trifolium hybridum .................. 5

$T$. repens ............................... 5 5

Trollius europaeus ...................... - 1

Tulipa sylvestris ....................... 11

Tussilago farfara ...................... $4 \quad 4 \quad 4$

Vaccinium uliginosum ............... 11

$V$. vitis-idaea ............................. $5 \quad 5$

Valeriana officinalis .................. 1 -

Varonica chamaedrys .................. - 1

V. longifolia ........................... 2 -

Viburnum lantana ..................... - 3

Vicia cracca ............................. 2 -

Viola tricolor

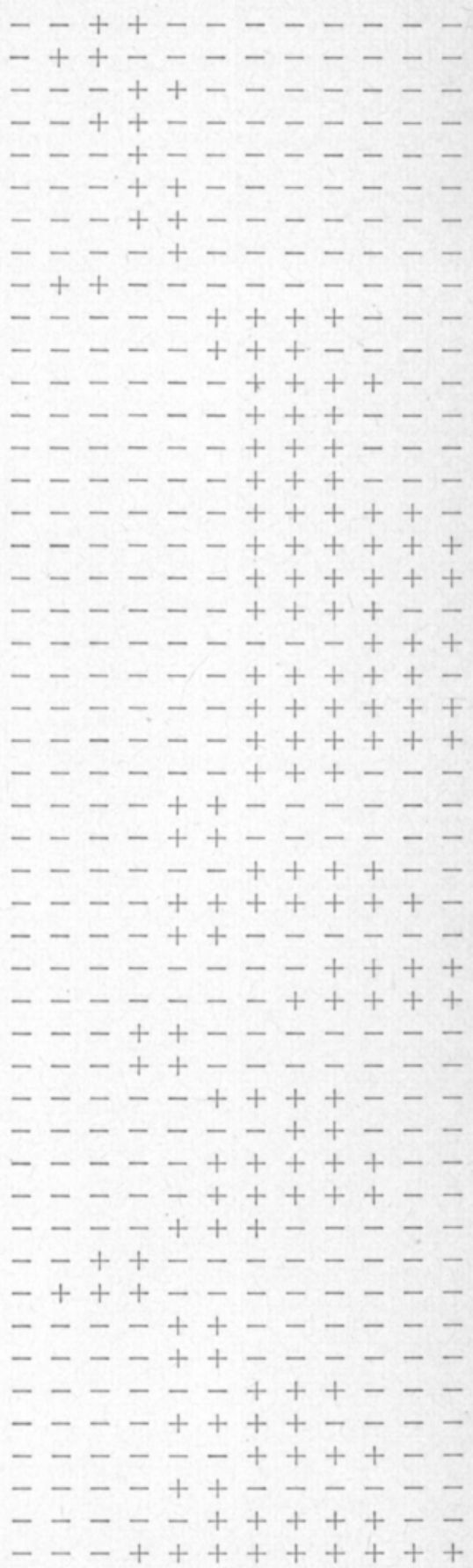


June or at the beginning of July. Most of the species belonging to this category have comparatively long flowering times and some continue flowering even to the end of the summer season. (4) Late summer and autumn species have their main flowering period at the end of July and in August. They continue their flowering also in September if the weather is warm enought.

The most important species visited during spring are Salix spp. and Tussilago farfara. During early summer other Salix species dominate, and others in the order of importance measured by the amount of visits observed are: Acer platanoides, Malus domestica, Taraxacum officinale, Vaccinium vitis-idaea. Arabis alpina, Barbarea vulgaris, Ribes uva-grispa, Prunus domestica, Sorbus aucuparia, Crataegus intricata, and Geranium sylvaticum. Among these Arabis alpina is a rather rare decorative plant. During mid-summer the most important species visited were Trifolium repens, T. hybridum, Rubus idaeus, Tilia cordata, Epilobium angustifolium, Frangula alnus, Cirsium arvense and Centaurea cyanus. During late summer and autumn the species mostly visited were Calluna vulgaris, Arctium tomentosum, Sedum spp., Sonchus arvensis and Leontodon autumnalis.

The most important honey plants in Finland are Trifolium repens and $T$. hybridum. Next in importance are probably Calluna vulgaris and Epilobium angustifolium, and in spring Salix spp. (cf. Martimo 1945, AARIo 1962, SARISALO and VALLE 1969).

\section{Discussion}

The amount of important honey plant species in Finland is much smaller than in Central Europe (cf. Maurizio and GrafL 1969), but all the species found important in Finland are also important in Central Europe.

The importance of a plant species as a food plant of Apis mellifera is first of all dependent on the nectar or pollen production of that species. Other important factors are the frequency and abundance of the species and its attractiveness to honey bees. The attractiveness is often affected by other plant species flowering at the same time. Thus the amount of visits that a particular flower species gets may vary very much from time to time and from place to place. Especially the amount of honey bees visiting red clover (Trifolium pratense) is very variable (VALLE 1959).

In spite of the relatively extensive data presented here, this list is not complete. Wind-pollinated species are almost totally absent, although honey bees are known to visit them for pollen (MAURIzIo and GRAFL 1969, HANSSON 1976). On one occasion I saw plenty of honey bees collecting pollen from Rumex acetosa.

Rumex pollen was also commonly found in AARIo's (1962) study. More observations are needed to ascertain the importance of wind-pollinated trees and grasses as pollen sources of honey bees in Finland. According to AARIo's (1962) data they are never or very rarely visited.

In this material there are no observations on visits to red clover (Trifolium pratense), which in fact is sometimes fairly frequently visited (e.g. VALLE 1959, Valle and Bergt 1965, Sarisalo and Valle 1969), Though as a whole its importance is small (AARIO 1962). 
Winter turnip rape (Brassica rapa var. oleifera), which for many years was locally an important food source, was not cultivated in Finland during the time of these field observations. Its cultivation became common, especially in southwestern Finland, from the year 1950 on wards (Hrrvola 1965). It is later replaced by annual forms of rape and turnip rape.

There are few observations concerning the nectar production and nectar quality of wild flowers in Finland (KÄPYLÄ 1978 b), although knowledge of the nectar yield of different vegetation types would be of great value in beekeeping.

\section{REFERENCES}

AArıo, R. 1962. Siitepōlytutkimuksia v. 1960 hunajasadosta Suomessa. Mehiläistalous 17: $61-63,76-78,93-95,121-123,150-153$.

Ehrendorfer, F. 1973. Liste der Gefässpflanzen Mitteleuropas. 318 p. Stuttgart.

Hansson, A. 1976. Biväxter. 111 p. Kristianstad.

Hirtonen, I. \& PoIJÄrvi, A. 1954. Koulu- ja retkeilykasvio. 8. painos. 472 p. Helsinki.

HıvolA, S.-L. 1965. Öljykasvitutkimukset Kasvinviljelylaitoksella 1942-1963. Summary: Investigations with oil crops at the Department of Plant Husbandry in 1942-1963. Acta Agr. Fenn. 107: 90-102.

KÄPYLÄ, M. 1978 a. Bionomics of five wood-nesting solitary species of bees (Hym., Megachilidae), with emphasis on flower relationships. Biol. Res. Rep. Univ. Jyväskylä 5: 3-89.

- 1978 b. Amount and type of nectar sugar in some wild flowers in Finland. Ann. Bot. Fenn. 15: $85-88$.

KUURMA, K. \& PAKarinen, L. 1951. Nykyaikainen mehiläishoito. 324 p. Helsinki.

Lönngvist, B. 1974. Pflanzenphänologische Beobachtungen in Finland 1961-1965. Bidr. Känn. Finl. Natur och Filk 112, 1: 1-47.

Mali, L. \& LaAksonen, K. J. 1961. Mehiläiskirja. 267 p. Helsinki.

Martimo, E. 1945. Suomalaisen hunajan ominaisuuksista ja alkuperästä. Referat: Uber die Zusammensetzung und Herkunft des finnischen Honigs. J. Scient. Agric. Soc. Finl. 17: $157-169$.

Maurizio, A. \& Grafl, I. 1969. Das Trachpflanzenbuch. 288 p. München.

Niemelä, P. 1934. Eteläsuomalaisten pikkumehiläisten kukillakäynneistä. 526 p. M.Sc. thesis, University of Turku.

Perttula, U. 1952. Metsäkasvien kukinta-ajoista ja niitten aiheuttamista värinäkymistä. Summary: On the blossoming periods and the colour aspects of forest flowers. Comm. Inst. For. Fenn. 40, 23: 1-25.

Reuter, M. 1948. Pflanzenphänologishe Beobachtungen in Finnland 1941-1945. Bird. Känn. Finl. Natur och Folk 92, 1: 1-79.

- 1952. Pflanzenphänologische Beobachtungen in Finnland 1946-1950. Bird. Känn. Finl. Natur och Folk 92, 3:1-64.

- 1957. Pflanzenphänologische Beobachtungen in Finland 1951-1955. Bird. Känn. Finl. Natur och Folk 100, 1: 1-63.

SARISALO. M. \& VALLE, O. 1969: Mehiläisrotujen työskentely puna-apilalla siitepölytutkimuksen perusteella. Summary: The activity of different races of honeybees on red clover on the basis of pollen studies. Acta Agr. Fenn. 113, 1: 1-34.

Tutin, T. G., Heywood, V. H., Burges, N. A., Valentine, D. H., Walters, S. H. \& WebB, D. A. 1954-1976. Flora europaea $1-4.464+456+370+505$ p. Cambridge.

Valle, K. J. 1951. Paavo Niemelä † Ann. Ent. Fenn. 17: 134-136.

VALlE, O. 1959. Kimalaiset ja mehiläiset puna-apilan pölyttäjinä. Summary: Bumble bees and honeybees as pollinators of red clover. Maatal. ja Koetoim. 13: 227-237.

- \& Bergt, K. 1965. Honeybees as pollen and nectar collectors in red clover. Ann. Acad. Sci. Fenn. A IV 91: 1-15.

Ms received December 15, 1978. 


\section{Mehiläisen ravintokasvit Etelä-Suomessa}

\section{MARKKU KÄPYLÄ}

Jyväskylän yliopisto, Biologian laitos, Yliopistonkatu 9, 40100 Jyväskylä 10.

\section{PaAvo Niemelä}

Kuollut 12. VII. 1951.

Tutkimus perustuu Paavo Niemelän vuosina 1931-1933 tekemiin laajoihin kenttähavaintoihin, käsittäen kaikkiaan 44500 havaintoa mehiläisen (Apis mellifera L.) kukilla käynneistä. Tästä aineistosta on koottu tavalliset kasvit (139 lajia) ja esitetty kukin lajin merkitys erikseen meden ja siitepölyn lähteenä. Lisäksi esitetään kasvien kukinta-ajat puolen kuukauden tarkkuudella.

Keväällä (huhti-toukokuussa) tärkeimmät ravintokasvit ovat pajut ja leskenlehti; alkukesällä (toukokuun lopussa ja kesäkuussa) pajut, voikukka, vaahtera, puolukka, kanankaali, karviainen, herukat, omenapuu, pihlaja ja metsäkurjenpolvi; keskikesällä valko- ja alsikeapila, vadelma, lehmus, maitohorsma, paatsama ja pelto-ohdake; loppukesällä ja alkusyksyllä kanerva, takiainen, peltovalvatti ja syysmaitiainen. 